\title{
SUMSETS OF MEASURABLE SETS
}

\author{
MELVYN B. NATHANSON
}

\begin{abstract}
Let $\mathbb{Q}_{1}, \mathbb{Q}_{2}, \ldots, \mathbb{Q}_{n}$ be Lebesgue measurable sets of positive real numbers such that inf $Q_{i}=0$ for all $i$. Let $\mu$ denote Lebesgue measure and let $\mu_{*}$ denote inner Lebesgue measure. If $\sum_{i-1}^{n} \mu\left(\mathbb{Q}_{i} \cap[0, t]\right) \gamma t$ for some $\gamma<1$ and all $t<x$, then

$$
\mu_{*}\left(\left(\mathbb{Q}_{1}+\mathbb{Q}_{2}+\cdots+\mathbb{Q}_{n}\right) \cap[0, x]\right)>\gamma x .
$$
\end{abstract}

This generalizes results of Dyson and Macbeath.

Let $A$ be a set of positive integers, and let $A(m)$ denote the number of elements of $A$ not exceeding $m$. The Schnirelmann density of $A$ is

$$
d(A)=\inf \{A(m) / m \mid m=1,2,3, \ldots\} .
$$

If $A$ and $B$ are sets of positive integers, then the sumset $A+B$ consists of all positive integers of the form $a+b$, where $a \in A \cup\{0\}$ and $b \in B \cup\{0\}$. Schnirelmann [12], [13] proved that, if $d(A)=\alpha$ and $d(B)=\beta$, then $d(A+$ $B) \geqslant \alpha+\beta-\alpha \beta$. Khinchin, Landau, and Schur conjectured that the stronger inequality $d(A+B) \geqslant \alpha+\beta$ should be true. After Besicovitch [2], Brauer [3], Khinchin [6], Landau [8], and Schur [14] obtained partial results, Mann [10] obtained an inequality that implied the $\alpha+\beta$ conjecture. Artin and Scherk [1] have given an exposition of Mann's proof.

ManN's Theorem. Let $A$ and $B$ be any sets of positive integers, and let $C=A+B$. Let $\gamma \leqslant 1$ be given such that

$$
A(m)+B(m) \geqslant \gamma m
$$

for $m=1,2, \ldots, g$. Then $C(g) \geqslant \gamma g$.

In 1945, Dyson [4] generalized Mann's Theorem to sums of any finite number of sets. Let $A_{1}, A_{2}, \ldots, A_{n}$ be sets of positive integers. Then the sumset $A_{1}+A_{2}+\cdots+A_{n}$ consists of all positive integers of the form $a_{1}+a_{2}+\cdots+a_{n}$, where $a_{i} \in A_{i} \cup\{0\}$ for $i=1,2, \ldots, n$. A sum of rank $r$ of the sets $A_{1}, A_{2}, \ldots, A_{n}$ is a sumset of the form $A_{i_{1}}+A_{i_{2}}+\cdots+A_{i_{1}}$, where $1 \leqslant i_{1}<i_{2}<\cdots<i_{r} \leqslant n$. The number of sums of rank $r$ is exactly $\left(\begin{array}{l}n \\ r\end{array}\right)$.

Received by the editors July 31, 1978.

AMS (MOS) subject classifications (1970). Primary 10L05; Secondary 28 A10.

Key words and phrases. Sums of sets of real numbers, sums of sequences, additive number theory, addition theorems.

${ }^{1}$ This research was supported in part by the National Science Foundation under grant MCS78-07908. 
Dyson's Theorem. Let $A_{1}, A_{2}, \ldots, A_{n}$ be $n$ sets of positive integers. For any pair of integers $r$ and $m$, let

$$
\phi_{r}(m)=\sum_{S} S(m)
$$

where the summation extends over the $\left(\begin{array}{c}n \\ r\end{array}\right)$ sums $S$ of rank $r$ of the sets $A_{1}, A_{2}, \ldots, A_{n}$. Let an integer $g$ and real number $\gamma \leqslant 1$ be given such that

$$
\phi_{1}(m)=A_{1}(m)+A_{2}(m)+\cdots+A_{n}(m) \geqslant \gamma m
$$

for $m=1,2, \ldots, g$. Then

$$
\phi_{r}(g) \geqslant\left(\begin{array}{l}
n-1 \\
r-1
\end{array}\right) \gamma g
$$

for $r=1,2, \ldots, n$. In particular, when $r=n$, so that $S=A_{1}+A_{2}$ $+\cdots+A_{n}$

$$
\phi_{n}(g)=S(g)>\gamma g .
$$

Halberstam and Roth [5], Khinchin [7], and Mann [11] have written excellent expositions of these results of additive number theory.

Let $\mathbb{Q}_{1}, \mathbb{Q}_{2}, \ldots, \mathbb{Q}_{n}$ be sets of real numbers. Then the sumset $\mathbb{Q}_{1}+\mathbb{Q}_{2}$ $+\cdots+Q_{n}$ consists of all numbers of the form $a_{1}+a_{2}+\cdots+a_{n}$, where $a_{i} \in \mathbb{Q}_{i}$ for $i=1,2, \ldots, n$. A sum of rank $r$ of the sets $\mathbb{Q}_{1}, \mathbb{Q}_{2}, \ldots, \mathbb{Q}_{n}$ is a sumset of the form $\mathbb{Q}_{i_{1}}+Q_{i_{2}}+\cdots+\mathbb{Q}_{i_{r}}$, where $1 \leqslant i_{1}<i_{2}<\cdots<i_{r}<$ $n$. Sierpinski [15] has proved that there exist Lebesgue measurable sets $\mathbb{Q}$ and $\mathscr{B}$ of real numbers whose sumset $\mathcal{Q}+\mathscr{B}$ is not measurable. Nonetheless, Macbeath [9] was able to obtain a continuous analog of Mann's Theorem for sums of two Lebesgue measurable sets of positive real numbers. Let $\mu$ denote Lebesgue measure and let $\mu_{*}$ denote inner Lebesgue measure.

MACbeath's Theorem. Let $\mathbb{Q}$ and $\mathscr{B}$ be Lebesgue measurable sets of positive real numbers such that inf $Q=\inf \mathscr{B}=0$, and let $\mathcal{C}=\mathbb{Q}+\Re$. If

$$
\mu(\mathscr{Q} \cap[0, t])+\mu(\mathscr{B} \cap[0, t]) \geqslant \gamma t
$$

for some $\gamma \leqslant 1$ and all $t \leqslant x$, then

$$
\mu_{*}(\bigodot \cap[0, x]) \geqslant \gamma x
$$

In this note I extend Macbeath's Theorem to sums of any finite number of Lebesgue measurable sets of positive real numbers. The proof follows Macbeath's proof very closely, but applies Dyson's Theorem instead of Mann's Theorem to obtain the result in the special case when all of the sets $\mathbb{Q}_{i}$ are open.

TheOREM. Let $\mathbb{Q}_{1}, \mathbb{Q}_{2}, \ldots, \mathbb{Q}_{n}$ be $n$ Lebesgue measurable sets of positive real numbers such that inf $\mathbb{Q}_{i}=0$ for $i=1,2, \ldots, n$. For any integer $r$ and real number $t$, let

$$
\Phi_{r}(t)=\sum_{\delta} \mu_{*}(\delta \cap[0, t])
$$


where the summation extends over the $\left(\begin{array}{l}n \\ r\end{array}\right)$ sums $\mathcal{S}$ of rank $r$ of the sets $\mathbb{Q}_{1}$, $\mathbb{Q}_{2}, \ldots, \mathbb{Q}_{n}$. Let real numbers $x$ and $\gamma \leqslant 1$ be given such that

$$
\Phi_{1}(t)=\mu\left(\mathbb{Q}_{1} \cap[0, t]\right)+\cdots+\mu\left(\mathbb{Q}_{n} \cap[0, t]\right)>\gamma t
$$

for all $t<x$. Then

$$
\Phi_{r}(x) \geqslant\left(\begin{array}{l}
n-1 \\
r-1
\end{array}\right) \gamma x
$$

for $r=1,2, \ldots, n$. In particular, when $r=n$, so that $\delta=\mathbb{Q}_{1}+\mathbb{Q}_{2}$ $+\cdots+Q_{n}$,

$$
\Phi_{n}(x)=\mu_{*}(\delta \cap[0, x]) \geqslant \gamma x .
$$

Proof. We can assume that $\mathbb{Q}_{i} \subseteq[0, x]$ for $i=1,2, \ldots, n$. Let us prove the theorem first in the case when all of the sets $\mathcal{Q}_{i}$ are open sets such that there exists $w>0$ such that $(0, w) \subseteq \mathbb{Q}_{i}$ for $i=1,2, \ldots, n$. For any $\delta>0$, let

$$
\mathbb{Q}_{i}^{\delta}=\left\{k \mid k \in \mathbf{Z} \text { and }((k-1) \delta, k \delta) \subseteq \mathbb{Q}_{i}\right\}
$$

and let

$$
A_{i}^{\delta}=\bigcup_{k \in A_{i}^{\delta}}((k-1) \delta, k \delta)
$$

Then $\mathbb{Q}_{i}^{\delta} \subseteq \mathbb{Q}_{i}$ and

$$
\mu\left(\mathbb{Q}_{i}^{\delta} \cap[0, m \delta]\right)=\delta A_{i}^{\delta}(m) .
$$

Moreover, if $m \leqslant g$, then

$$
\begin{aligned}
\mu\left(\mathbb{Q}_{i} \cap[0, m \delta]\right)-\mu\left(\mathbb{Q}_{i}^{\delta} \cap[0, m \delta]\right) \\
<\mu\left(\mathbb{Q}_{i} \cap[0, g \delta]\right)-\mu\left(\mathbb{Q}_{i}^{\delta} \cap[0, g \delta]\right) .
\end{aligned}
$$

Since the sets $\mathscr{Q}_{i}$ are open and bounded, for any $\varepsilon>0$ there exists $\delta_{0}>0$ such that, if $\delta<\delta_{0}$, then $\mu\left(\mathbb{Q}_{i}^{\delta}\right)>\mu\left(\mathbb{Q}_{i}\right)-\varepsilon$ for $i=1,2, \ldots, n$.

Let $\mathcal{S}=\mathbb{Q}_{i_{1}}+\mathbb{Q}_{i_{2}}+\cdots+\mathbb{Q}_{i_{r}}$ be a sum of rank $r$ of the sets $\mathbb{Q}_{1}, \mathbb{Q}_{2}, \ldots, \mathbb{Q}_{n}$. Since the sets $\mathbb{Q}_{i}$ are open, if $J \subseteq\{1,2, \ldots, r\}$, then

$$
\sum_{j \in J} \mathbb{Q}_{i_{j}} \subseteq \sum_{j=1}^{r} \mathbb{Q}_{i_{j}}=\delta .
$$

For $\delta>0$, let $S^{\delta}=\{k \mid k \in \mathbf{Z}$ and $((k-1) \delta, k \delta) \subseteq \delta\}$. I shall show that

$$
S=A_{i_{1}}^{\delta}+A_{i_{2}}^{\delta}+\cdots+A_{i_{r}}^{\delta} \subseteq s^{\delta} \text {. }
$$

Let $s=a_{1}+a_{2}+\cdots+a_{r} \in S$, where $a_{j} \in A_{i j}^{\delta} \cup\{0\}$ for $j=1,2, \ldots, r$ and $a_{j} \neq 0$ for some $j$. Let $J=\left\{j \mid a_{j} \neq 0\right\}$. Since $a_{j} \in A_{i j}^{\delta}$ for $j \in J$, it follows that $\left(\left(a_{j}-1\right) \delta, a_{j} \delta\right) \subseteq \mathcal{Q}_{i}$, and so

$$
\begin{aligned}
((s-1) \delta, s \delta) & \subseteq\left(\sum_{j \in J}\left(a_{j}-1\right) \delta, \sum_{j \in J} a_{j} \delta\right) \\
& \subseteq \sum_{j \in J} \mathbb{Q}_{i_{j}} \subseteq \delta .
\end{aligned}
$$

Therefore, $s \in S^{\delta}$. This proves (1). 
Fix $\varepsilon>0$. Let $\delta=x / g$, where the integer $g$ is chosen sufficiently large that

$$
\mu\left(\mathbb{Q}_{i}^{\delta}\right)>\mu\left(\mathbb{Q}_{i}\right)-w \varepsilon / n
$$

for $i=1,2, \ldots, n$. Let $m \leqslant g$ and let $t=m \delta$. Suppose $t \geqslant w$. Then

$$
\begin{aligned}
\delta \sum_{i=1}^{n} A_{i}^{\delta}(m)= & \sum_{i=1}^{n} \mu\left(\mathbb{Q}_{i}^{\delta} \cap[0, m \delta]\right)=\sum_{i=1}^{n} \mu\left(\mathbb{Q}_{i} \cap[0, m \delta]\right) \\
& -\sum_{i=1}^{n}\left\{\mu\left(\mathbb{Q}_{i} \cap[0, m \delta]\right)-\mu\left(\mathbb{Q}_{i}^{\delta} \cap[0, m \delta]\right)\right\} \\
\geqslant & \sum_{i=1}^{n} \mu\left(\mathbb{Q}_{i} \cap[0, t]\right)-\sum_{i=1}^{n}\left\{\mu\left(\mathbb{Q}_{i} \cap[0, g \delta]\right)-\mu\left(\mathbb{Q}_{i}^{\delta} \cap[0, g \delta]\right)\right\} \\
> & \sum_{i=1}^{n} \mu\left(\mathbb{Q}_{i} \cap[0, t]\right)-w \varepsilon \\
\geqslant & \gamma t-\varepsilon t=(\gamma-\varepsilon) m \delta .
\end{aligned}
$$

Therefore,

$$
\sum_{i=1}^{n} A_{i}^{\delta}(m)>(\gamma-\varepsilon) m
$$

if $t=m \delta>w$. If $t<w$, then $(0, t) \subseteq(0, w) \subseteq \mathbb{Q}_{i}$ and so $\{1,2, \ldots, m\} \subseteq A_{i}^{\delta}$ and $A_{i}^{\delta}(m)=m$. Thus, (2) also holds for $t<w$, and so (2) holds for $m=1$, $2, \ldots, g$. By Dyson's Theorem,

$$
\phi_{r}(g)=\sum_{S} S(g) \geqslant\left(\begin{array}{l}
n-1 \\
r-1
\end{array}\right)(\gamma-\varepsilon) g
$$

where the summation extends over all sums $S$ of rank $r$ of the sets $A_{1}^{\delta}$, $A_{2}^{\delta}, \ldots, A_{n}^{\delta}$.

Let $S=A_{i_{1}}^{\delta}+A_{i_{2}}^{\delta}+\cdots+A_{i_{r}}^{\delta}$ be one of these sums of rank $r$, and let $\delta=\mathbb{Q}_{i_{1}}+\mathbb{Q}_{i_{2}}+\cdots+\mathbb{Q}_{i_{r}}$ be the corresponding sum of rank $r$ of the open sets $\mathbb{Q}_{1}, \mathbb{Q}_{2}, \ldots, \mathbb{Q}_{n}$. Let $S^{\delta}=\{k \mid k \in \mathbf{Z}$ and $((k-1) \delta, k \delta) \subseteq \delta\}$. I showed above that $S \subseteq S^{\delta}$. Therefore,

$$
\sum_{\delta} S^{\delta}(g) \geqslant \sum_{S} S(g) \geqslant\left(\begin{array}{l}
n-1 \\
r-1
\end{array}\right)(\gamma-\varepsilon) g
$$

where the first summation extends over all sums $\delta$ of rank $r$ of the open sets $\mathbb{Q}_{1}, \mathbb{Q}_{2}, \ldots, \mathbb{Q}_{n}$. Therefore, since $x=g \delta$,

$$
\begin{aligned}
\Phi_{r}(x) & =\sum_{\delta} \mu(\delta \cap[0, x]) \geqslant \delta \sum_{\delta} S^{\delta}(g) \\
& >\left(\begin{array}{l}
n-1 \\
r-1
\end{array}\right)(\gamma-\varepsilon) g \delta=\left(\begin{array}{l}
n-1 \\
r-1
\end{array}\right)(\gamma-\varepsilon) x .
\end{aligned}
$$

The inequality holds for all $\varepsilon>0$, hence

$$
\Phi_{r}(x) \geqslant\left(\begin{array}{l}
n-1 \\
r-1
\end{array}\right) \gamma x .
$$

This proves the theorem when the $\mathbb{Q}_{i}$ are open sets and $(0, w) \subseteq \mathbb{Q}_{i}$. The extension of the theorem, first to the case in which the sets $\mathbb{Q}_{i}$ are closed, and then to the case of arbitrary Lebesgue measurable sets $Q_{i}$, follows exactly as in Macbeath's proof. 
It is an open problem to generalize Mann's Theorem and Dyson's Theorem to sums of sets of finite-dimensional vectors with nonnegative integral coordinates, and to generalize Macbeath's Theorem to sums of Lebesgue measurable sets in finite-dimensional Euclidean spaces.

\section{REFERENCES}

1. E. Artin and P. Scherk, On the sum of two sets of integers, Ann. of Math. (2) 44 (1943), $138-142$.

2. A. Besicovitch, On the density of the sum of two sequences of integers, J. London Math. Soc. 10 (1935), 246-248.

3. A. Brauer, On the density of the sum of sets of positive integers. II, Ann. of Math. (2) 42 (1941), 959-988.

4. F. Dyson, A theorem on the densities of sets of integers, J. London Math. Soc. 20 (1945), 8-14.

5. H. Halberstam and K. F. Roth, Sequences, Oxford Univ. Press, Oxford, 1966.

6. A. Y. Khinchin, Zur additiven Zahlentheorie, Mat. Sb. 39 (1932), 27-34.

7. _ Three pearls of number theory, Graylock, Rochester, New York, 1952.

8. E. Landau, Die Goldbachsche Vermutung und der Schnirelmannsche Satz, Gottinger Nachr., Math. Phys. K1. (1930), 255-276.

9. A. M. Macbeath, On measure of sumsets. III. The continuous $(\alpha+\beta)$-theorem, Proc. Edinburgh Math. Soc. (2) 12 (1960/61), 209-211; correction, ibid. 14 (1964/65), 165-166.

10. H. B. Mann, A proof of the fundamental theorem on the density of sums of sets of positive integers, Ann. of Math. (2) 43 (1942), 523-527.

11. __ Addition theorems, Wiley, New York, 1965.

12. L. G. Schnirelmann, On additive properties of numbers, Izv. Politekh. Inst. Novocherkasske 14 (1930), 3-27; reprinted in Uspehi Mat. Nauk 6 (1939), 9-25.

13. __ Uber additive Eigenschaften von Zahlen, Math. Ann. 107 (1933), 649-690.

14. I. Schur, Uber den Begriff der Dichte in der additiven Zahlentheorie, S.-B. Preuss. Akad. Wiss. Math. Phys. K1. (1936), 269-297.

15. W. Sierpinski, Sur la question de la mesurabilité de la base de M. Hamel, Fund. Math. 1 (1920), 105-111.

Department of Mathematics, Harvard University, Cambridge, Massachusetts 02138

Current address: Department of Mathematics, Southern Illinois University, Carbondale, Illinois 62901 